\title{
Stem cell division is regulated by the microRNA pathway
}

\author{
S. D. Hatfield ${ }^{1 \star}$, H. R. Shcherbata ${ }^{1 \star}$, K. A. Fischer ${ }^{1}$, K. Nakahara ${ }^{2}$, R. W. Carthew ${ }^{2} \&$ H. Ruohola-Baker ${ }^{1}$
}

One of the key characteristics of stem cells is their capacity to divide for long periods of time in an environment where most of the cells are quiescent. Therefore, a critical question in stem cell biology is how stem cells escape cell division stop signals. Here, we report the necessity of the microRNA (miRNA) pathway ${ }^{1-4}$ for proper control of germline stem cell (GSC) division in Drosophila melanogaster. Analysis of GSCs mutant for dicer-1 (dcr-1), the double-stranded RNaseIII essential for miRNA biogenesis, revealed a marked reduction in the rate of germline cyst production. These $d c r-1$ mutant GSCs exhibit normal identity but are defective in cell cycle control. On the basis of cell cycle markers and genetic interactions, we conclude that $d c r-1$ mutant GSCs are delayed in the G1 to $S$ transition, which is dependent on the cyclindependent kinase inhibitor Dacapo, suggesting that miRNAs are required for stem cells to bypass the normal G1/S checkpoint. Hence, the miRNA pathway might be part of a mechanism that makes stem cells insensitive to environmental signals that normally stop the cell cycle at the G1/S transition.

MicroRNAs and short interfering RNAs (siRNAs), processed by the type III double-stranded RNase Dicer, function in an RNA-based mechanism of gene silencing ${ }^{1-4}$. Most characterized miRNAs from animals repress gene expression by blocking the translation of complementary messenger RNAs into protein; they interact with their targets by imperfect base-pairing to mRNA sequences within the $3^{\prime}$ untranslated region $\left(3^{\prime} \text { UTR }\right)^{1}$. Experimental evidence has suggested that small RNAs regulate stem cell character in plants and animals $s^{5-7}$. Moreover, some miRNAs are differentially expressed in stem cells, suggesting a specialized role in stem cell regulation ${ }^{8,9}$. However, the molecular mechanisms underlying stem cell control by miRNAs are not understood.

To determine the role of miRNAs in the control of stem cell biology, we specifically eliminated processing of all miRNAs in stem cells. The Drosophila genome contains two Dicer isozymes: Dicer-1 and Dicer-2 (ref. 10). Dicer-1 (Dcr-1) is essential for processing miRNAs, whereas Dicer-2 (Dcr-2) is required for siRNAs; loss of Dcr-1 completely disrupts the miRNA pathway and only has a weak effect on the siRNA pathway. Using Drosophila GSCs as a model system, we impaired Dcr-1 activity with two $d c r-1$ alleles: $d c r-1^{d 102}$ and a null $d c r-1^{\text {Q1147X }}$ (ref. 10). Drosophila oogenesis depends on the presence of self-renewing GSCs in the adult ovary ${ }^{11,12}$, as has recently been reported in a mammalian system ${ }^{13}$. The continuous division of GSCs generates an array of progressively developed egg chambers in wild-type ovarioles (Fig. 1).

Analysis of $d c r-1$ mutant clones in the Drosophila ovary 12 days after clone induction revealed a marked depletion of developing egg chambers (see Fig. 1b-f for $d c r-1^{\text {Q1147X }}$ and Supplementary Fig. 1a

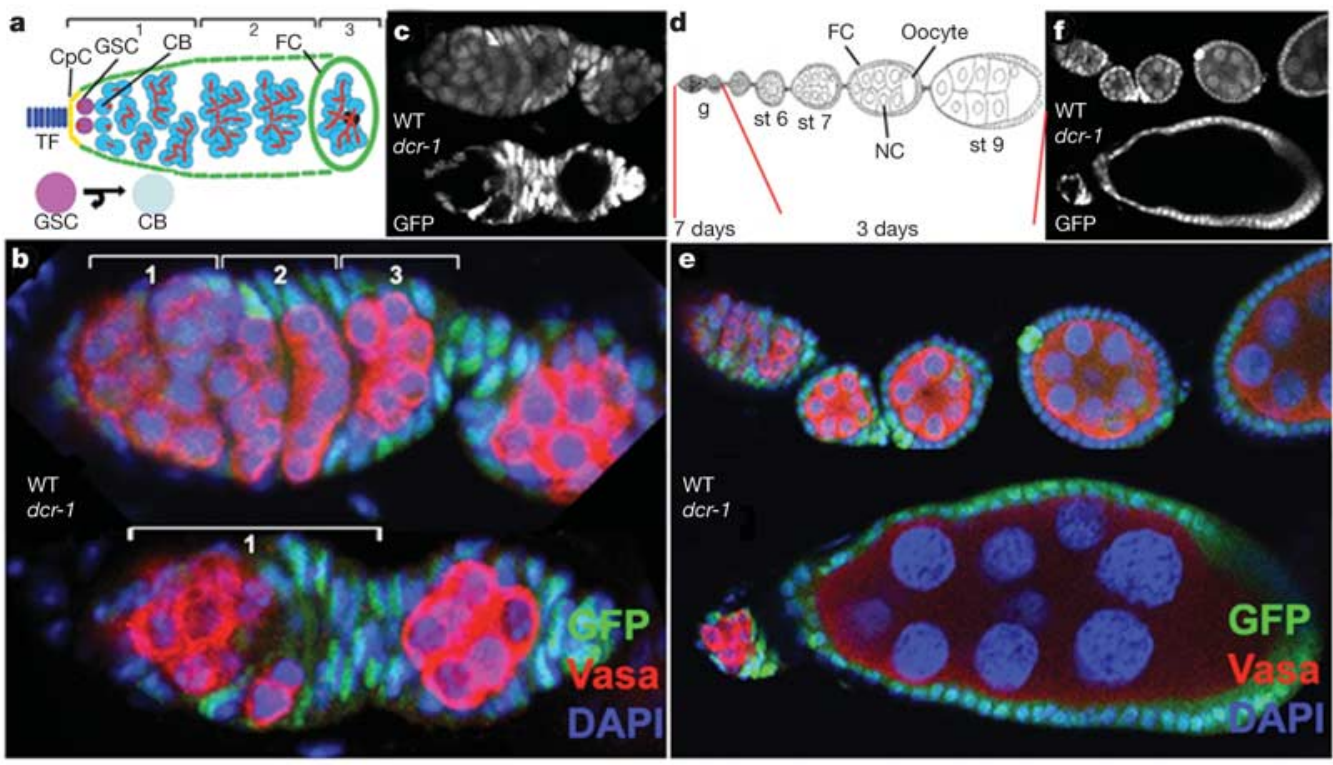

Figure 1 | Loss of Dcr-1 function in GSCs reduces the rate of egg chamber production.

a, Schematic of a germarium divided into three regions. Region one contains GSCs and dividing cysts. b, c, All three regions are observed in a wild-type heterozygous $d c r-1^{\mathrm{Q} 1147 \mathrm{X}} /+$ germarium (WT, top), but not in a mosaic dcr-1 ${ }^{\text {Q1147X }}$ germarium 12 days after clone induction (no GFP, $d c r$-1, bottom). d, Oocyte development is divided into 14 stages. e, f, Many of the developmental stages are missing in ovarioles that are complete $d c r-1^{\text {Q1147X }}$ germline clones (no GFP, 12 days after clone induction; bottom of panels e and f). $\mathrm{CpC}$, cap cells; $\mathrm{CB}$, cystoblast; DAPI, 4,6-diamidino-2phenylindole; FC, follicle cells; NC; nurse cells; TF, terminal filaments. Vasa marks the germ line. Absence of GFP marks dcr-1 mutant cells.

${ }^{1}$ Department of Biochemistry, University of Washington, J591, HSB, Seattle, Washington 98195-7350, USA. ²Department of Biochemistry, Molecular Biology and Cell Biology, Northwestern University, 2205 Tech Drive, Evanston, Illinois 60208, USA.

*These authors contributed equally to this work. 
for $\left.d c r-1^{d 102}\right)$. In contrast, $d c r-2$ null mutant GSCs produced a normal progression of egg chambers. These data suggest that Dcr-1 is required for efficient germline production. Although $d c r-1$ mutants showed reduced numbers of gametes, most developing gametes appeared morphologically normal (although they exhibit polarity defects; data not shown). We therefore analysed potential problems in GSC maintenance, identity and division. Clonal experiments revealed that the percentage of germaria with clonal stem cells at different time points after clone induction was similar in the $d c r-1^{\text {Q1147X }}$ mutant and the wild-type control (Fig. 2b), suggesting that the loss of cysts in $d c r-1$ mutants is not primarily due to problems in the maintenance of GSCs.

To determine whether reduced cyst production in $d c r-1$ germaria was due to altered GSC fate, we analysed the identity of the $d c r-1$ mutant GSCs. Female GSCs are identified by their location and the expression patterns of three markers (Fig. 2a): the presence of Adducin, a protein present in the spectrosome ${ }^{14}$; the presence of phosphorylated Mad protein (P-Mad), indicating TGF- $\beta$ signalling $^{14,15}$; and the absence of Bam, repressed by the TGF- $\beta$ pathway ${ }^{16}$. The $d c r-1{ }^{\text {Q1147X }}$ GSCs showed normal spectrosome morphology and position $(100 \%, n=53)$, and normal TGF- $\beta$ pathway activity (P-Mad: wild type $88 \%, n=114$; $d c r-1^{\text {Q1147X }} 85 \%, n=47$; Fig. $\left.2 c, d\right)$. Furthermore, as with wild-type GSCs, $d c r-1^{\text {Q1147X }}$ GSCs did not stain positively for the Bam protein (Fig. 2e). From these analyses, we conclude that decreased cyst production from $d c r-1^{\text {Q1147X }}$ GSCs does not result from either a loss of GSCs or a change in their identity.

The frequency of cell division in $d c r-1^{\text {Q1147X }}$ GSCs was impaired. Examination of individual germaria containing a single heterozygous GSC and a single $d c r-1^{\text {Q1147X }}$ mutant GSC revealed that GSCs lacking Dcr-1 activity produced cysts at a frequency that was reduced to $18 \%$ of normal levels ( $41 \%$ for $d c r-1^{d 102}$; Table 1 and Fig. $3 \mathrm{a}-\mathrm{c}$ ). In contrast, the frequency of division was not significantly reduced for GSCs that were homozygous for the $d c r-2$ mutation or for the isogenized parental chromosome from which the $d c r-1$ mutant alleles were generated (Table 1 and Fig. 3c). Thus, Dcr-1 is required cell autonomously in GSCs for the cell divisions that produce developing cystoblasts (no obvious defect in cyst division was observed; see Supplementary Fig. 2c, d).

To determine whether the reduced cyst formation reflected a block in the normal cell cycle programme, we analysed the distribution of cell cycle stages in mutant $d c r-1^{\text {Q1147X }}$ GSCs by staining mosaic germaria with antibodies against different cell cycle markers (Fig. 3d).

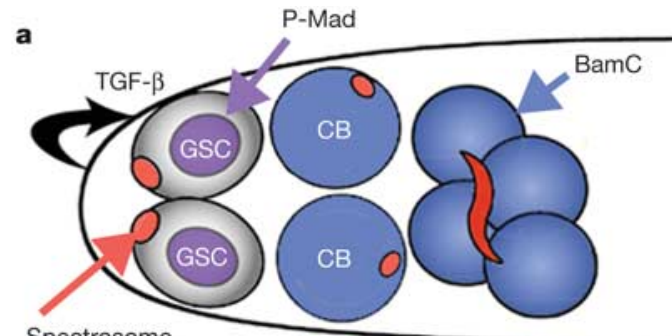

Spectrosome
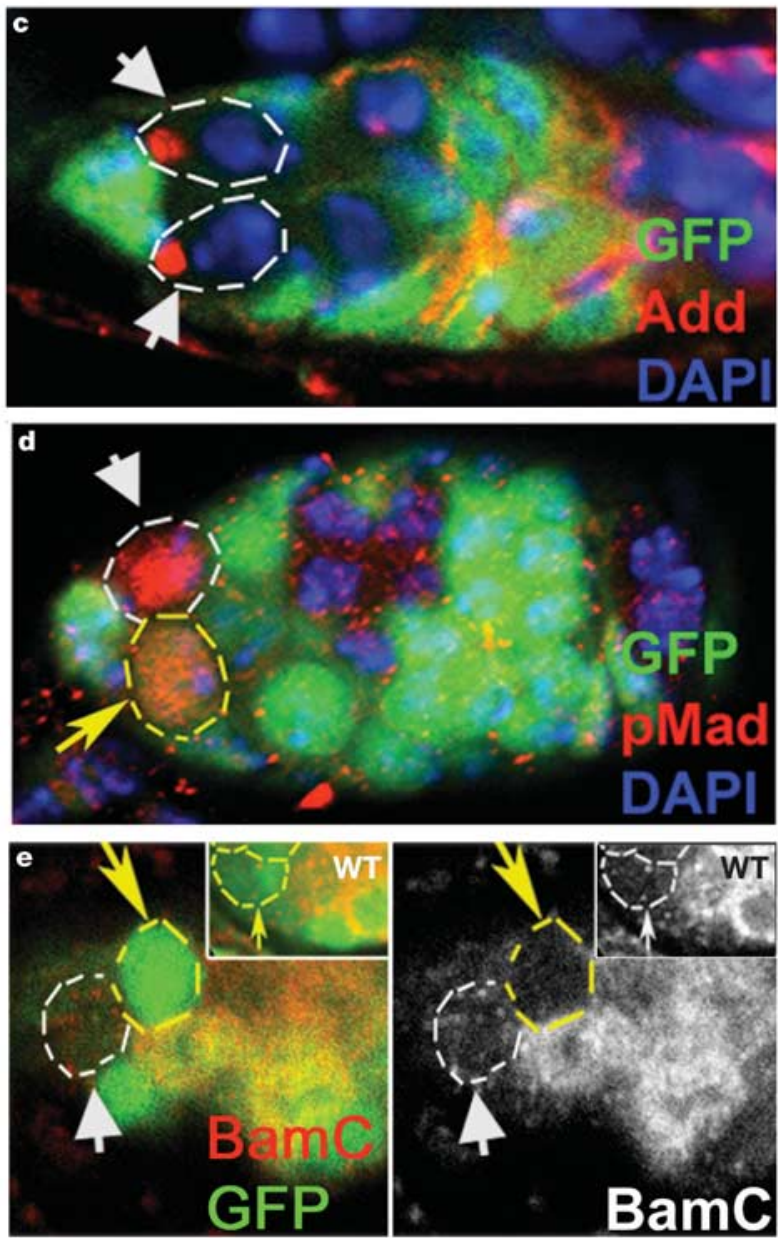

b
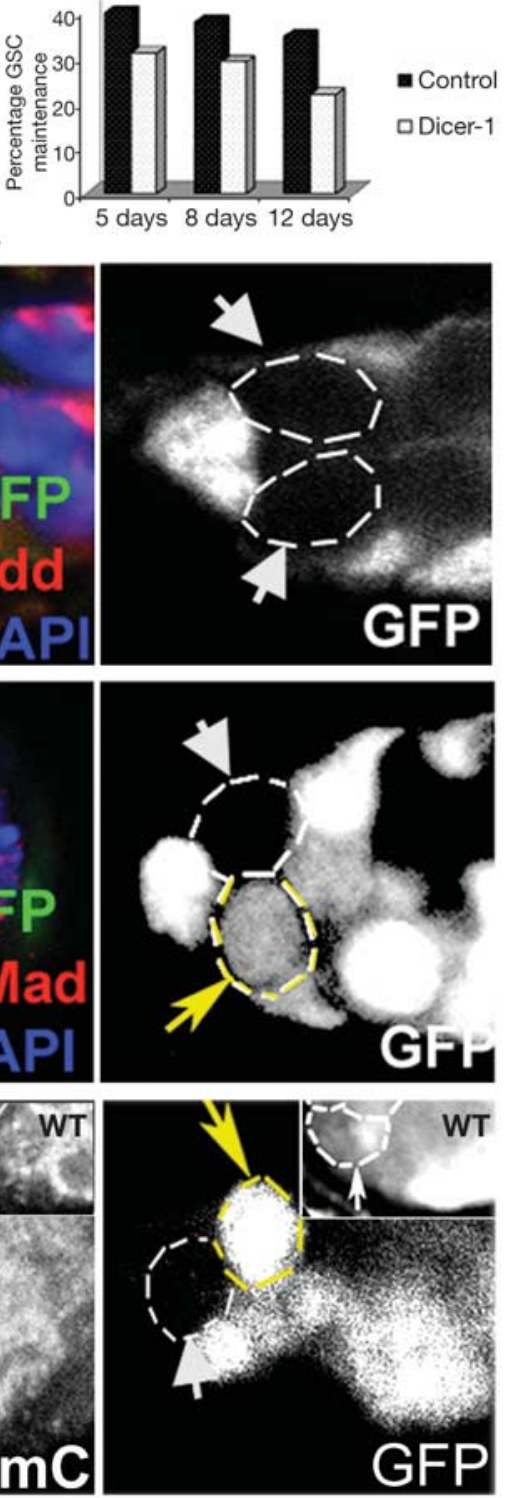

Figure $2 \mid$ dcr-1 mutant GSCs remain in the stem cell niche and retain stem cell identity. a, GSC markers: P-Mad, spectrosome (Adducin, Add), lack of BamC. $\mathbf{b}, d c r-1^{\text {Q1147X }}$ mutant and wildtype GSCs are maintained in germaria. A low rate of GSC loss was observed for both backgrounds ${ }^{30}$. Bars represent the percentage of germaria with clonal stem cells at different time points after clone induction. c-e, $d c r-1^{\text {Q1147X }}$ GSCs (no GFP, white arrows) possess a spectrosome $(100 \%, n=53$; c) and respond to the TGF- $\beta$ signal (anti-P-Mad staining: $d c r-1$ mutant, $85 \%, n=47$; control, $88 \%, n=114$; d) but do not exhibit BamC expression $(n=13$; e). Yellow arrows in d, e mark non-clonal, wild-type GSCs. 
Table 1 | Effect of the miRNA pathway on GSC division frequency

\begin{tabular}{|c|c|c|c|c|}
\hline Brief genotype & Genotype of GSC and cysts & $\begin{array}{c}\text { Frequency of GSC division } \\
( \pm \text { s.d. })^{\star}\end{array}$ & $\begin{array}{l}\text { Sample size } \\
(n)\end{array}$ & $\begin{array}{l}\text { Division index } \\
( \pm \text { s.d. }) \dagger\end{array}$ \\
\hline Wild type & Clonal (GFP-'): hsFlp; FRT82B hsNmyc/FRT82B hsNmyc & $4.64 \pm 0.87$ & 17 & $1.03 \pm 0.20$ \\
\hline$d c r-10^{01147 x}$ & Non-clonal $\left(\mathrm{GFP}^{+}\right)$: hsFlp; FRT82B dcr-1 11147X /FRT82B Ubi-GFP & $4.37 \pm 0.74$ & 27 & - \\
\hline$d c r-7^{Q 1147 X}$ & Clonal (GFP-): hsFlp; FRT82B dcr-1 ${ }^{\text {Q1147X }} /$ FRT82B dcr-1 ${ }^{\text {Q1147X }}$ (12 days) & $0.77 \pm 0.59$ & 20 & $0.18 \pm 0.13$ \\
\hline$d c r-1^{d 102}$ & Non-clonal $\left(\mathrm{GFP}^{+}\right)$: hsFlp; FRT82B dcr-1 1102 /FRT82B Ubi-GFP & $4.64 \pm 0.83$ & 24 & - \\
\hline$d c r-1^{d 102}$ & Clonal $\left(\mathrm{GFP}^{-}\right)$: hsFlp; FRT82B dcr-1 ${ }^{1102} /$ FRT $82 B d c r-1^{d 102}$ ( 8 days) & $2.5 \pm 0.65$ & 24 & $0.53 \pm 0.26$ \\
\hline$d c r-1^{d 102}$ & Clonal $\left(\mathrm{GFP}^{-}\right)$: hsFlp; FRT82B dcr-1 ${ }^{d 102} /$ FRT82B dcr-1 ${ }^{d 102}$ (12 days) & $1.72 \pm 0.56$ & 39 & $0.41 \pm 0.13$ \\
\hline$d c r-2$ & Clonal (GFP $\left.{ }^{-}\right)$: hsFIp; FRT42D dcr-2/FRT42D dcr-2 (12 days) & $4.20 \pm 1.36$ & 24 & $0.85 \pm 0.28$ \\
\hline Parental chromosome & Non-clonal $\left(G^{\prime} P^{+}\right)$: hsFlp; FRT82B (parental)/FRT82B Ubi-GFP & $4.58 \pm 0.97$ & 23 & - \\
\hline Parental chromosome & Clonal (GFP'): hsFlp; FRT82B (parental)/FRT82B (parental) (8 days) & $4.80 \pm 1.04$ & 23 & $1.04 \pm 0.17$ \\
\hline Parental chromosome & Clonal $\left(\mathrm{GFP}^{-}\right)$: hsFlp; FRT82B (parental)/FRT82B (parental) (12 days) & $5.77 \pm 0.69$ & 22 & $1.16 \pm 0.14$ \\
\hline dap & Non-clonal $\left(\mathrm{GFP}^{+}\right)$: hsFlp; FRT42B dap ${ }^{4} /$ FRT $42 B$ Ubi-GFP & $4.44 \pm 0.66$ & 20 & - \\
\hline dap & Clonal $\left(\mathrm{GFP}^{-}\right)$: hsFlp; FRT42B dap ${ }^{4} /$ FRT $42 B$ dap ${ }^{4}$ & $4.12 \pm 0.79$ & 20 & $0.93 \pm 0.18$ \\
\hline$d a p /+; d c r-1$ & 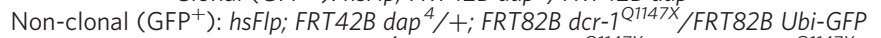 & $4.25 \pm 0.55$ & 29 & - \\
\hline
\end{tabular}

8 or 12 days indicate the number of days after clone induction (this is 8 days if not indicated).

${ }^{\star}$ The number of cystoblasts and cysts divided by the number of GSCs.

$\uparrow$ The frequency of clonal GFP- GSC division divided by the frequency of control non-clonal GFP ${ }^{+} \mathrm{GSC}^{-}$division.

We observed an increase in the number of $d c r-1$ mutant GSCs staining positive for Cyclin $\mathrm{E}(\mathrm{CycE})$ using two independent $d c r-1$ alleles (Fig. 3e, f; see also Supplementary Table 1 and Supplementary Fig. 1b). In contrast, GSCs that were homozygous for $d c r-2$ or the parental chromosome expressed CycE with frequencies similar to that of wild-type GSCs (Supplementary Fig. 1b). Furthermore, pulse labelling of ovaries with the nucleotide analogue 5-bromodeoxyuridine $(\mathrm{BrdU})^{17,18}$ revealed that the number of $d c r-1^{\mathrm{Q} 1147 \mathrm{X}}$
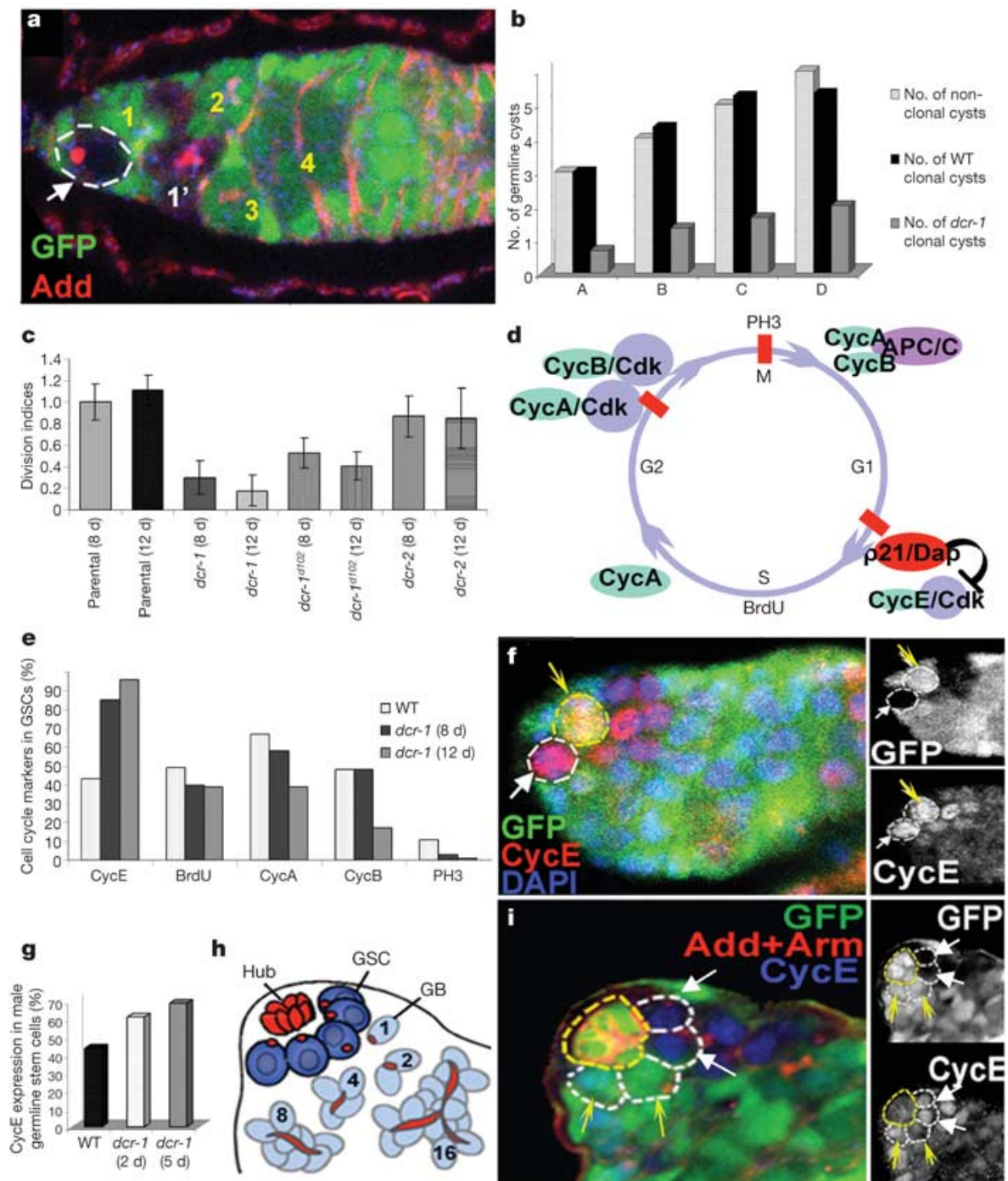
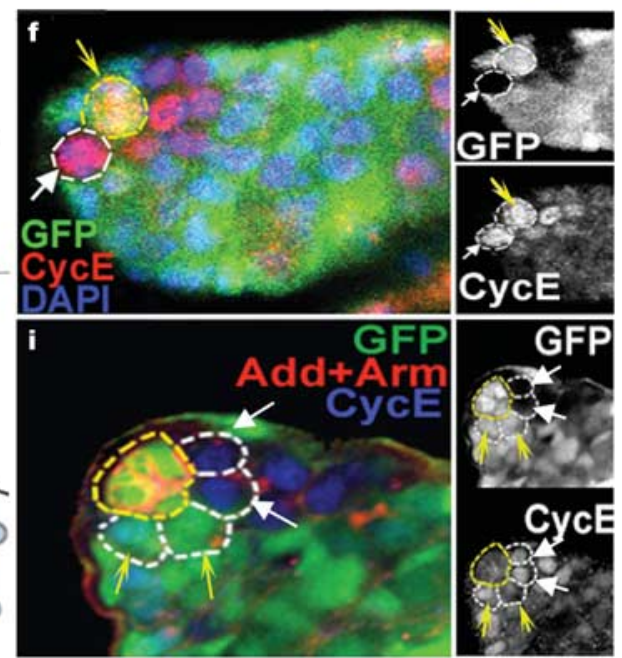

GFP

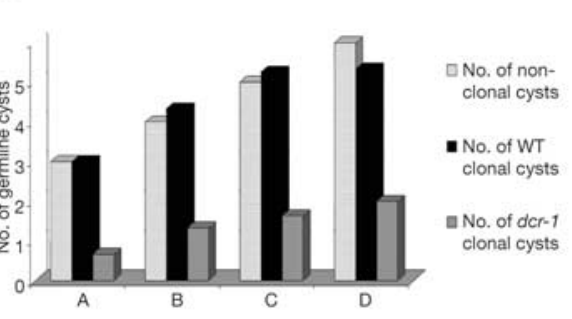

d

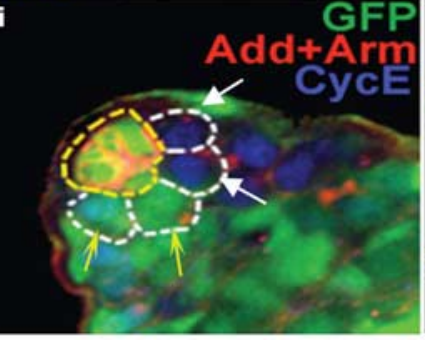

Figure 3 |dcr-1 causes a cell cycle delay in GSCs. a, $d c r-1{ }^{\text {Q1147X }}$ GSCs (white arrow) produce fewer cysts (white number) than wildtype GSCs (yellow numbers). b, $d c r-1^{\text {Q1147X }}$ cysts are produced at a lower frequency than wild-type and non-clonal cysts. Samples A-D are grouped according to the number of non-clonal cysts per germarium $(n=27)$. c, Division indices are decreased for $d c r-1$ mutant GSCs compared with control and $d c r-2$ mutant GSC clones. Error bars represent standard deviation. Times are days after the last clonal induction. d, Cell cycle markers. e, $d c r-1^{\text {Q1147X }}(d c r-1)$ GSCs are more frequently CycE-positive and less frequently positive for other cell cycle markers compared with wild-type $d c r-1^{\mathrm{Q} 1147 \mathrm{X}} /+$ GSCs. f, CycEpositive $d c r-1$ mutant GSC (white arrow). g, Percentage of CycE-positive male GSCs that are $d c r-1^{\mathrm{Q} 1147 X}$ or wild type. h, Male GSC niche. i, CycE in male $d c r-1$ mutant GSCs (white arrow). Arm (Armadillo) marks somatic hub cells. Yellow arrows in $\mathbf{f}$, $\mathbf{i}$ mark non-clonal, wild-type GSCs. 
mutant GSCs in S phase was reduced (Fig. 3e; see also Supplementary Table 1). Similarly, the number of $d c r-1^{\text {Q1147X }}$ mutant GSCs staining positive for Cyclin A (CycA), Cyclin B ( $\mathrm{CycB}$ ) and the mitotic marker Phosphohistone-3 (PH3) was reduced (Fig. 3e; see also Supplementary Table 1). These data indicate that perturbation of the miRNA pathway by mutant $d c r-1$ in GSCs delays the cell cycle at the G1/S transition.

We tested whether loss of Dcr-1 function has similar consequences on the cell cycle in the GSCs of male flies. Each male testis contains approximately ten GSCs surrounding a somatic structure called the

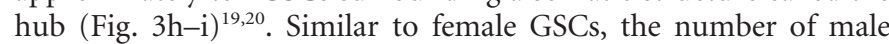
GSCs staining positive for $\mathrm{CycE}$ was increased in $d c r-1$ mutants (Fig. $3 \mathrm{~g}-\mathrm{i}$ ). These data show that Dcr-1 also functions in the male GSC niche, and suggest that Dcr-1 has a conserved role in GSC division.

To test the possibility that the miRNA pathway might be a general cell cycle regulator, we examined other cell types to determine whether the G1/S delay and reduced cell division frequency are also observed in other mitotically dividing $d c r-1$ mutant cells. $d_{c r-1}{ }^{\text {Q1147X }}$ clones in imaginal discs revealed that the number of CycE-positive cells was not increased in mutant cells (Supplementary Fig. 2a). The number of $d c r-1^{\text {Q1147X }}$ mutant cells in imaginal discs was approximately equal to the number of marked wild-type cells that descended from a common parent cell, indicating that the frequency of cell division in imaginal disc cells is not reduced in a $d c r-1$ mutant (Supplementary Fig. 2a, b). $d c r-1{ }^{\text {Q1147X }}$ dividing germline cysts express CycE at a frequency comparable to that of wild-type dividing cysts, suggesting that the mitotic cystoblast cell divisions are not affected in $d c r-1$ mutants (Supplementary Fig. 2c, d). Therefore, the reduction in cell division frequency observed in the $d c r-1$ mutant germ line is specific to the GSC division. Together, these data suggest that the miRNA pathway has a specific role in regulating stem cell division.

We explored the potential cause for the G1/S arrest by examining the expression of Dacapo (Dap; a homologue of the p21/p27 family of cyclin-dependent kinase (CDK) inhibitors) ${ }^{21,25}$ in $d c r-1^{\text {Q1147X }}$ mutant GSCs. The transition between the G1 and S phases of the cell cycle is negatively regulated by $\mathrm{Dap}^{21,25}$. Dap protein traps the CycE/CDK2 complex in a stable but inactive form ${ }^{22}$, and elevated levels of Dap lead to cell cycle arrest at the G1/S phase transition with prolonged expression of CycE protein ${ }^{17}$. Notably, the number of Dap-positive GSCs increased in the $d c r-1$ mutant GSC population (Fig. 4b; see also Supplementary Table 1 and Supplementary Fig. 3a).

To determine whether Dap mediated the effect of $d c r-1$ on the GSC cell cycle, we reduced the level of Dap by $50 \%$ in $d c r-1{ }^{\text {Q1147X }}$ mutant GSCs and observed a partial rescue in cyst production (Table 1 and Fig. 4c, e). Furthermore, the number of GSCs staining positive for CycE was reduced to normal levels (Fig. 4d), demonstrating that the CycE defect observed in $d c r-1$ mutant GSCs is dependent on Dap. Consistent with this, overexpression of a Dap transgene resulted in some germaria resembling $d c r-1$ germline mutants: the germaria were small, containing a few cysts, and had a high number of CycEpositive GSCs (Fig. 4d; see also Supplementary Fig. 3d). The fact that reduction of Dap levels led to a normal GSC CycE profile, but partial rescue of cyst generation, suggests that Dcr-1 might also regulate later cyst development.

These data suggest that miRNAs act on stem cell division by reducing the levels of Dap. How is this regulation achieved? We found

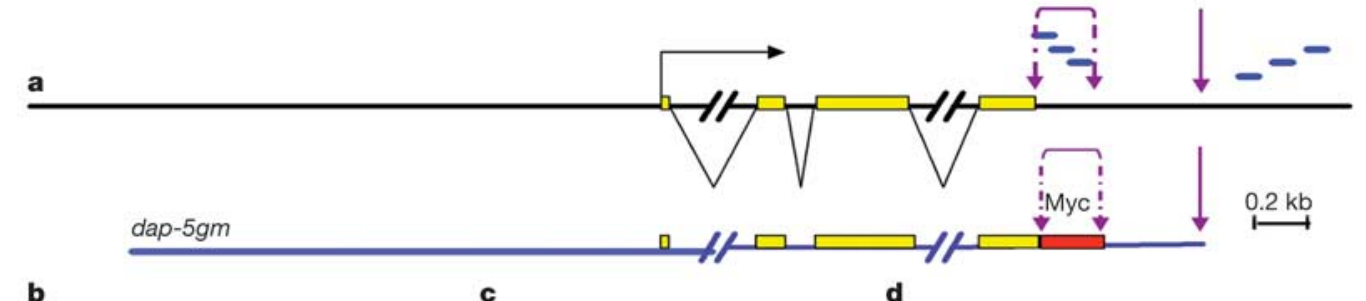

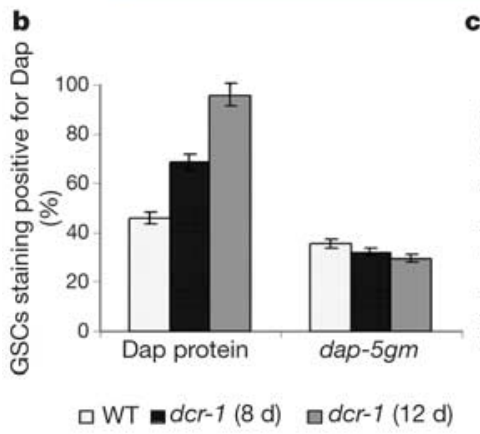

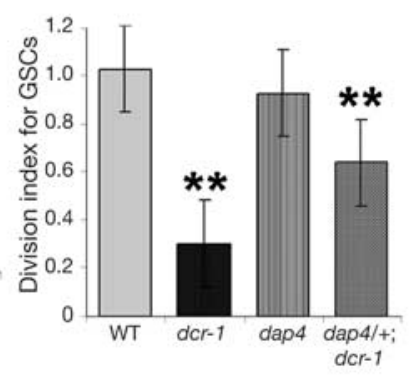

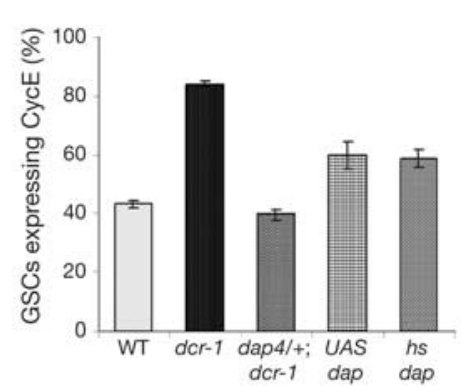

$\mathbf{f}$

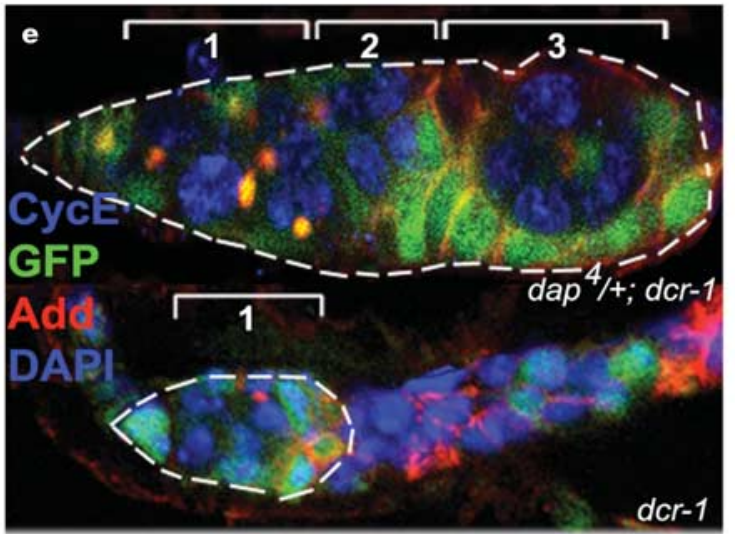

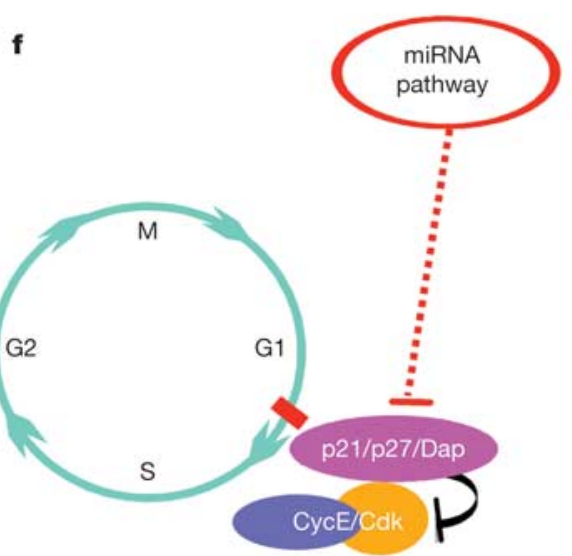

Figure 4 | The GSC division defect is dependent on Dap. $a$, The dap-5gm transgene compared to the endogenous dap locus. A $6 \times$ Myc tag replaces a region of the 3 ' UTR containing predicted miRNA-binding sites (blue bars). The UTR lacks the last 540 base pairs. b, Endogenous Dap is detected more frequently in $d c r-1$ mutant than wild-type GSCs but Dap from dap-5gm is not detected more frequently in $d c r-1$ GSCs.

c, e, Germaria with dap ${ }^{4} /+; d c r-11^{\text {Q1147X }}$ GSCs produce more cysts than germaria with $d c r-1^{\text {Q1147X }}$ GSCs. Double asterisk, $P \leq 10^{-3}$ (Student's $t$-test). d, Percentage of CycE-positive GSCs. Dap was overexpressed by nos-Gal4/pUASp-dap or hs-dap. Error bars represent standard deviations (c) or errors from the mean (b, d). f, miRNA pathway modulates the GSC cell cycle by affecting the G1/S transition through Dap (p21/p27). 
that expression of a Dap transgene containing the Dap promoter and essentially all of the endogenous gene except some of the $3^{\prime}$ UTR $(\text { dap- } 5 \mathrm{gm})^{23}$ was similar in $d c r-1$ mutant and wild-type GSCs (Fig. 4b; see also Supplementary Fig. 3b, c). These data suggest that the effect of Dcr-1 on Dap regulation in GSCs (Fig. 4b) is at a post-transcriptional level and might involve the $3^{\prime}$ UTR region that is missing in the dap-5gm transgene (Fig. 4a).

We propose that miRNAs are required for GSCs to transit the G1/S checkpoint by repressing directly or indirectly the G1/S inhibitor Dap (Fig. 4f). Because Dap is a key component of the G1/S transition ${ }^{21-25}$, it is a plausible target for machinery that assures continuous cell division in a microenvironment in which most of the cells are quiescent. We propose that while the TGF- $\beta$ pathway-which can upregulate p21/p27 (ref. 24) -is active in GSCs ${ }^{16}$, miRNAs downregulate Dap to assure the continuous cell division essential for stem cells. This downregulation might be direct, because the Dap 3' UTR contains several predicted miRNA-binding sites ${ }^{26-28}$ (Supplementary Fig. 4). A Dap transgene lacking these sites showed no response to Dcr-1 levels (Fig. 4b; see also Supplementary Fig. 3b, c), suggesting that the potential binding sites are responsive to Dicer-1. However, it is also possible that the Dap misregulation in $d c r-1$ mutant GSCs might be due to a secondary effect of Dcr-1 loss. Our finding that miRNAs are required for stem cell division suggests that miRNAs might be part of a mechanism that makes stem cells insensitive to environmental signals that normally stop the cell cycle. Because miRNAs are a novel class of genes involved in human tumorigenesis ${ }^{29}$, it is tempting to speculate that miRNAs could have a similar role in cancer cells.

\section{METHODS}

We used the following stocks: eyFLP;FRT82Bdcr-1 ${ }^{\text {Q1147X }} / T M 3 S b$, eyFLP;FRT82Bdcr-1 ${ }^{d 102} / T M 3 S b$, eyFLP;FRT42Ddcr- $2^{L 811 X} / C y O$, eyFLP;FRT82B parental ${ }^{10}, w ; F R T 42 B d a p^{4} / C y O$ (ref. 25), dap5gm (ref. 23), w;NGT40/SM6a; nosGal4VP16/TM3Sb, hsFlp;FRT82BUbi-GFP/TM3Sb, hsFlp;FRT42BUbi-GFP/ CyO, hsFlp;FRT42DUbi-GFP/CyO, FRT82BhsNmyc.

For female germline clones, Flp-FRT flies were heat shocked (third instar larvae for $1 \mathrm{~h}$, pupae for $1 \mathrm{~h}$ at $37^{\circ} \mathrm{C}$ ) and dissected 5-12 days after the last heat shock. For male germline clones, adult flies were heat shocked for $40 \mathrm{~min}$ at $37^{\circ} \mathrm{C}$ twice per day for 3 days and dissected 2-6 days after the last heat shock.

We used the following antibodies: mouse anti-CycA, anti-CycB, anti-Adducin (1:20) and anti-Armadillo (1:40) (Developmental Studies Hybridoma Bank), mouse anti-Dap (1:5, I. Hariharan), anti-BrdU (1:20, PharMingen) and anti-cMyc (1:50, Calbiochem), rabbit anti-PH3 (1:200, Upstate Biotechnology), antiGFP (1:1,000, Molecular Probes), anti-Vasa (1:10,000, P. Lasko) and anti-P-Mad (1:500, P. ten Dijke), guinea-pig anti-CycE (1:500, T. Orr-Weaver), rat antiBamC (1:1,000, D. McKearin), Alexa 488, 568, or 633 goat anti-mouse, antirabbit and anti-guinea-pig (1:500, Molecular Probes), and goat-anti-rat Cy5 (1:250, Jackson Immunoresearch).

\section{Received 23 March; accepted 16 May 2005}

\section{Published online 8 June 2005}

1. He, L. \& Hannon, G. J. MicroRNAs: small RNAs with a big role in gene regulation. Nature Rev. Genet. 5, 522-531 (2004)

2. Nakahara, K.\& Carthew, R. W. Expanding roles for miRNAs and siRNAs in cell regulation. Curr. Opin. Cell Biol. 16, 127-133 (2004)

3. Bartel, B. \& Bartel, D. P. MicroRNAs: at the root of plant development? Plant Physiol. 132, 709-717 (2003).

4. Ambros, V. microRNAs: tiny regulators with great potential. Cell 107, 823-826 (2001).

5. Bernstein, E. et al. Dicer is essential for mouse development. Nature Genet. 35 215-217 (2003).

6. Schauer, S. E., Jacobsen, S. E., Meinke, D. W. \& Ray, A. DICER-LIKE1: blind men and elephants in Arabidopsis development. Trends Plant Sci. 7, 487-491 (2002)

7. Carmell, M. A., Xuan, Z., Zhang, M. Q. \& Hannon, G. J. The Argonaute family: tentacles that reach into RNAi, developmental control, stem cell maintenance, and tumorigenesis. Genes Dev. 16, 2733-2742 (2002).
8. Suh, M. R. et al. Human embryonic stem cells express a unique set of microRNAs. Dev. Biol. 270, 488-498 (2004).

9. Houbaviy, H. B., Murray, M. F. \& Sharp, P. A. Embryonic stem cell-specific MicroRNAs. Dev. Cell 5, 351-358 (2003)

10. Lee, Y. S. et al. Distinct roles for Drosophila Dicer-1 and Dicer-2 in the siRNA/ miRNA silencing pathways. Cell 117, 69-81 (2004)

11. Spradling, A., Drummond-Barbosa, D. \& Kai, T. Stem cells find their niche. Nature 414, 98-104 (2001).

12. Gilboa, L. \& Lehmann, R. How different is Venus from Mars? The genetics of germ-line stem cells in Drosophila females and males. Development 131, 4895-4905 (2004).

13. Johnson, J., Canning, J., Kaneko, T., Pru, J. K. \& Tilly, J. L. Germline stem cells and follicular renewal in the postnatal mammalian ovary. Nature 428, 145-150 (2004).

14. Kai, T.\& Spradling, A. Differentiating germ cells can revert into functional stem cells in Drosophila melanogaster ovaries. Nature 428, 564-569 (2004)

15. Newfeld, S. J. et al. Mothers against dpp participates in a DDP/TGF- $\beta$ responsive serine-threonine kinase signal transduction cascade. Development 124, 3167-3176 (1997).

16. Song, $X$. et al. Bmp signals from niche cells directly repress transcription of a differentiation-promoting gene, bag of marbles, in germline stem cells in the Drosophila ovary. Development 131, 1353-1364 (2004)

17. Shcherbata, H. R., Althauser, C., Findley, S. D. \& Ruohola-Baker, H. The mitotic to-endocycle switch in Drosophila follicle cells is executed by Notch-dependent regulation of G1/S, G2/M and M/G1 cell-cycle transitions. Development 131, 3169-3181 (2004).

18. Calvi, B. R. \& Lilly, M. A. Fluorescent BrdU labeling and nuclear flow sorting of the Drosophila ovary. Methods Mol. Biol. 247, 203-213 (2004).

19. Gonczy, P. \& DiNardo, S. The germ line regulates somatic cyst cell proliferation and fate during Drosophila spermatogenesis. Development 122, 2437-2447 (1996).

20. Kiger, A. A., Jones, D. L., Schulz, C., Rogers, M. B. \& Fuller, M. T. Stem cell selfrenewal specified by JAK-STAT activation in response to a support cell cue. Science 294, 2542-2545 (2001).

21. de Nooij, J. C., Letendre, M. A. \& Hariharan, I. K. A cyclin-dependent kinase inhibitor, Dacapo, is necessary for timely exit from the cell cycle during Drosophila embryogenesis. Cell 87, 1237-1247 (1996).

22. Pavletich, N. P. Mechanisms of cyclin-dependent kinase regulation: structures of Cdks, their cyclin activators, and Cip and INK4 inhibitors. J. Mol. Biol. 287, 821-828 (1999)

23. Meyer, C. A. et al. Drosophila p27Dacapo expression during embryogenesis is controlled by a complex regulatory region independent of cell cycle progression. Development 129, 319-328 (2002)

24. Datto, M. B. et al. Transforming growth factor beta induces the cyclindependent kinase inhibitor p21 through a p53-independent mechanism. Proc Natl Acad. Sci. USA 92, 5545-5549 (1995).

25. Lane, M. E. et al. Dacapo, a cyclin-dependent kinase inhibitor, stops cell proliferation during Drosophila development. Cell 87, 1225-1235 (1996).

26. Enright, A. J. et al. MicroRNA targets in Drosophila. Genome Biol. 5, R1 (2003).

27. Stark, A., Brennecke, J., Russell, R. B. \& Cohen, S. M. Identification of Drosophila MicroRNA targets. PLoS Biol. 1, E60 (2003).

28. Lewis, B. P., Shih, I. H., Jones-Rhoades, M. W., Bartel, D. P. \& Burge, C. B. Prediction of mammalian microRNA targets. Cell 115, 787-798 (2003).

29. He, L. et al. A microRNA polycistron as a potential human oncogene. Nature doi:10.1038/nature03552 (in the press).

30. Margolis, J. \& Spradling, A. Identification and behavior of epithelial stem cells in the Drosophila ovary. Development 121, 3797-3807 (1995).

Supplementary Information is linked to the online version of the paper at www.nature.com/nature.

Acknowledgements We thank B. Calvi, B. Wakimoto and E. Ward for comments on the manuscript, and the members of the H.R.-B. laboratory for suggestions throughout the course of this study. We also thank E. Kerr for making dacapo constructs; V. Shcherbatyy for creating transgenic lines; B. Akiyashi for UAS-Dap experiments; K. Kim for the $d c r-1^{d 102}$ strain; and M. Lilly, C. Lehner, I. Hariharan, T. Orr-Weaver, D. McKearin, P. ten Dijke, A. Spradling and C.-H. Heldin for flies, antibodies and advice. This work was supported by the Schultz Fellowship for S.D.H., grants from the National Institutes of Health to R.W.C and H. R.-B., and the American Heart Association and the American Cancer Society to H.R.-B.

Author Information Reprints and permissions information is available at npg.nature.com/reprintsandpermissions. The authors declare no competing financial interests. Correspondence and requests for materials should be addressed to H.R.-B. (hannele@u.washington.edu) and R.W.C. (r-carthew@northwestern.edu). 